\title{
Verzeichnis der Mitglieder der Vereinigung der Deutschen Staatsrechtslehrer
}

\author{
Stand: Januar 1979
}

Vorstand

1. Stern, Dr. Klaus, o. Professor, Am Stockberger Busch, 5067 Kürten, Tel. (02283) 6167

2. Oppermann, Dr. Dr. h. c. Thomas, o. Professor, Burgholzweg 122, 7400 Tübingen, Tel. (07071) 23762

3. Scholz, Dr. Rupert, o. Professor, Prof.-Huber-Platz 2, 8000 München 22, Tel. (089) 21802113

Mitglieder

1. Abelein, Dr. Manfred, o. Professor, Rheinweg 12 , 5300 Bonn, Tel. (02221) 25692

(Universität Regensburg)

2. Abendroth, Dr. Wolfgang, Professor, Neuhauss Str. 5, 6000 Frankfurt/M.

3. Achterberg, Dr. Norbert, o. Professor, Schweriner Str. 15, 4400 Münster, Tel. (02534) 7422

4. Adamovich, Dr. Ludwig, o. Professor a. D., Universitätsdozent, Roosevelt-Platz 4, A 1090 Wien, Tel. (0222) 427395 (Universität Graz)

5. Antoniolli, Dr. Dr. h. c. Walter, o. Universitätsprofessor, Ottensteinstr. 35, A 2344 Maria Enzersdorf, Tel. (02236) 4509 (Universität Wien)

6. Armbruster, Dr. Hubert, o. Professor, An der Allee 69, 6500 Mainz, Tel. (06131) 31950

7. Arndt, Dr. Hans-Wolfgang, Privatdozent, TheodorHaubach-Str. 4, 6200 Wiesbaden, Tel. (06121) 464974 (Universität Mainz)

8. v. Arnim, Dr. Hans Herbert, Professor, Emser Str. 39, 6200 Wiesbaden, Tel. (06121) 404213 (Universität Marburg)

9. Arnold, Dr. Rainer, o. Professor, Wolfsteinerstr. 14, 8400 Regensburg, Tel. (0941) 99670

10. Baade, Dr. Hans W., Professor, 6002 Mountain Climb Drive, Austin/Texas, USA. 78 731, Tel. (512) 452-5071 und 471-5151 (University of Texas) 
11. Bachof, Dr. Dr. h. c. Otto, o. Professor, Auf dem Kreuz 3, 7400 Tübingen, Tel. (07071) 61144

12. Badura, Dr. Peter, o. Professor, Habsburger Str. 2, 8000 München 40, Tel. (089) 342457

13. Barbey, Dr. Günther, Privatdozent, Stallupöner Allee 22, 1000 Berlin 19, Tel. (030) 3055703

14. Barfuß, Dr. jur. Dr. rer. pol. Walter, ao. Professor, Tegetthoffstr. 3, A 1010 Wien, Tel. (0222) 516841

15. Bartlsperger, Dr. Richard, o. Professor, Schleifweg 55, 8521 Uttenreuth, Tel. (09131) 59916

(Universität Erlangen)

16. Bäumlin, Dr. Richard, o. Professor, Riedmaad, CH 3765 Oberwil i. S. (Universität Bern)

17. Battis, Dr. Ulrich, Wiss. Rat und Professor, Dorfstr. 16, 2059 Wangelau, Tel. (04155) 3935 (Universität Hamburg)

18. Bayer, Dr. Hermann-Wilfried, Wiss. Rat und Professor, Nußbaumweg 25, 4630 Bochum 7, Tel. (0234) 715724

19. Becker, Dr. jur. Dr. phil. Erich, o. Professor, Philipp-Melanchthon-Str. 10, 6720 Speyer, Tel. (06232) 76439

20. Berchtold, Dr. Klaus, Universitätsdozent, Bräunerstr. 4-6/22, A 1010 Wien, Tel. (0222) 531434

21. Berg, Dr. Wilfried, Wiss. Rat und Professor, Südostring 101, 4401 Havixbeck, Tel. (02507) 7701 (Universität Münster)

22. Bernhardt, Dr. Rudolf, Professor, Gustav-Kirchhoff-Str. 2a, 6900 Heidelberg, Tel. (06221) 43699

23. Bethge, Dr. Herbert, o. Professor, Am Seidenhof 10, 8390 Passau, Tel. (0851) 41697

24. Bettermann, Dr. Karl August, o. Professor, Alte Landstr. 173, 2000 Hamburg 63, Tel. (040) 5384064

25. Bleckmann, Dr. Dr. Albert, o. Professor, Krummer Timpen 20, 4403 Nordwalde, Tel. (02573) 704 (Universität Münster)

26. Blümel, Dr. Willi, o. Professor, Angelhofweg 65, 6901 Wilhelmsfeld, Tel. (06220) 1880 (Hochschule Speyer)

27. Blumenwitz, Dr. Dieter, o. Professor, Herzog-Albrecht-Str. 26, 8011 Zorneding, Tel. (08106) 2682 (Universität Würzburg) 
28. Böckenförde, Dr. jur. Dr. phil. Ernst-Wolfgang, o. Professor, Türkheimstr. 1, $7801 \mathrm{Au}$ bei Freiburg, Tel. (0761) 405623

29. Böckstiegel, Dr. Karl-Heinz, o. Professor, Thywissenstr. 27, 4040 Neuss, Tel. (02101) 400279 (Universität Köln)

30. Bogs, Dr. Harald, o. Professor, Dresdener Str. 7, 3406 Bovenden, Tel. (0551) 81595 (Universität Göttingen)

31. Bothe, Dr. Michael, apl. Professor, Kurlandstr. 1, 6906 Leimen 3, Tel. (06224) 3560 (Universität Heidelberg)

32. Breuer, Dr. Rüdiger, Wiss. Rat und Professor, Stegeisen 6, 4800 Bielefeld 15, Tel. (05206) 3651

33. Brohm, Dr. Winfried, o. Professor, Wydenmöslistr. 11, CH 8280 Kreuzlingen, Tel. (072) 751525 (Universität Konstanz)

34. Brünner, Dr. Christian, ao. Universitätsprofessor, Rosengasse 10, A 8042 Graz, Tel. (03122) 418573

35. Brunner, Dr. Georg, o. Professor, Sonnleite 3, 8702 Lengfeld üb. Würzburg, Tel. (0931) 271636 (Universität Göttingen)

36. Bülck, Dr. Hartwig, o. Professor, Hohe Roth, 6121 Schöllenbach (Hochschule Speyer)

37. Bull, Dr. Hans Peter, o. Professor, Stephan-Lochner-Str. 2, 5300 Bonn 2, Tel. (02221) 3750 91-98 (Universität Hamburg)

38. Bullinger, Dr. Martin, o. Professor, Altschlößleweg 4, $7801 \mathrm{Au}$ bei Freiburg, Tel. (0761) 402389

39. Burmeister, Dr. Joachim, o. Professor, Am Hügel 11, 6600 Saarbrücken, Tel. (0681) 37328

40. Frhr. v. Campenhausen, Dr. Axel, o. Professor, Grünewaldstr. 6, 3000 Hannover 1, Tel. (0511) 691581

41. Carstens, Dr. Karl, o. Professor, Dechant-Kreiten-Str. 43, 5309 Meckenheim, Tel. (02225) 2455

42. Dagtoglou, Dr. Prodromos, o. Professor, Hippokrates Str. 33, Athen 144 (dienstl.)

43. Delbrück, Dr. Jost, o. Professor, Schoolredder 20, 2300 Kiel 16, Tel. (0431) 322558 
44. Denninger, Dr. Erhard, o. Professor, Am Wiesenhof 1, 6240 Königstein 3, Tel. (06173) 78932 (Universität Frankfurt)

45. Dicke, Dr. Detlev Christian, Professor, Alpenstr. 919, CH 3178 Bösingen (Universität Freiburg/Schweiz)

46. Doehring, Dr. Karl, o. Professor, Bergstr. 58, 6900 Heidelberg, Tel. (06221) 45880

47. Dreier, Dr. Ralf, o. Professor, Wilhelm-Weber-Str. 4-6, 3400 Göttingen, Tel. (0551) 59114

48. Dürig, Dr. Günter, o. Professor, Staufenstr. 9, 7400 Tübingen 9, Tel. (07071) 82508

49. Ehmke, Dr. Horst, o. Professor, Konrad-Adenauer-Str. 19, 5340 Rhöndorf/Rhein, Tel. (02224) 6108

50. Eichenberger, Dr. Kurt, o. Professor, Bärenbrunnenweg 4, CH 4144 Arlesheim bei Basel, Tel. (061) 723386

51. Erbel, Dr. Günter, Professor, Endenicher Allee 5, 5300 Bonn, Tel. (02221) 635124

52. Erichsen, Dr. Hans-Uwe, o. Professor, Frans-Hals-Str. 37 4630 Bochum 1, Tel. (0234) 432970

53. Erler, Dr. Georg, o. Professor, Charlottenburger Str. 19, 3400 Göttingen-Geismar

54. Ermacora, Dr. Felix, o. Universitätsprofessor, Karl-Lueger-Ring, A 1010 Wien I, Universität, Tel. (0222) 427611

55. Evers, Dr. Hans-Ulrich, o. Universitätsprofessor, Wolfsgartenweg 30, A 5020 Salzburg, Tel. (06222) 260203

56. Faber, Dr. Heiko, o. Professor, Wunstorfer Str. 1, 3007 Gehrden 1, Tel. (0511) 7628206 (Technische Universität Hannover)

57. Fiedler, Dr. Wilfried, Professor, Schillerstr. 16, $2300 \mathrm{Kiel}$, Tel. (0431) 8 80-21 89 (dienstl.)

58. Fleiner, Dr. Thomas, o. Professor, Le Riedelet 9, CH 1723 Marly FR, Tel. (037) 461261 (Universität Freiburg/Schweiz)

59. Folz, Dr. Hans-Ernst, o. Universitätsprofessor, Wolf-Huber-Str. 2, A 4020 Linz, Tel. (07222) 348594

60. Friauf, Dr. Karl Heinrich, o. Professor, Eichenhainallee 17, 5060 Bensberg-Frankenforst, Tel. (02204) 61984 (Universität Köln) 
61. Friesenhahn, Dr. Dr. h. c. Ernst, o. Professor, Wegeler Str. 2, 5300 Bonn, Tel. (02221) 657538

62. Fröhler, Dr. Ludwig, o. Universitätsprofessor, Altenberger Str. 39, A 4010 Linz-Urfahr

63. Fromont, Dr. Michel, Professeur de droit public, 9, Impasse Henri Bouchard, F 21000 Dijon, Tel. (80) 434808

64. Frotscher, Dr. Werner, o. Professor, Hundersinger Str. 11, 7000 Stuttgart 70, Tel. (0711) 455711 (Universität Hohenheim)

65. Frowein, Dr. Jochen A., o. Professor, Hobergerfeld 9, 4800 Bielefeld 1, Tel. (0521) 102311

66. Funk, Dr. Bernd-Christian, o. Professor, Ramperstorffer Gasse 2/12, A 1050 Wien, Tel. (0222) 575226 (Universität Graz)

67. Fuß, Dr. Ernst-Werner, o. Professor, Küsterbergstr. 8, 8702 Zell am Main, Tel. (0931) 463116 (Universität Würzburg)

68. Gallwas, Dr. Hans-Ulrich, Wiss. Rat und Professor, Hans-Leipelt-Str. 16, 8000 München 40, Tel. (089) 328366

69. Geck, Dr. Wilhelm Karl, M. A., o. Professor, 6671 Oberwürzbach-Reichenbrunn, Tel. (06894) 7326 (Universität des Saarlandes)

70. Gerber, D. Dr. Hans, o. Professor, In der Röte 5, 7800 Freiburg/Br., Tel. (0761) 53306

71. Göldner, Dr. Detlef, Privatdozent, Melanchthonstr. 21, 7400 Tübingen, Tel. (07071) 292545 (dienstl.)

72. Görg, Dr. Hubert, Professor, 5062 HoffnungsthalEllersberg, Tel. (02205) 2540 (Universität Marburg)

73. Götz, Dr. Volkmar, o. Professor, Nikolausberger Weg 56, 3400 Göttingen, Tel. (0551) 43119

74. Grabitz, Dr. Eberhard, o. Professor, Up de Schanz 30, 2000 Hamburg 52, Tel. (040) 828060 (Freie Universität Berlin)

75. Grawert, Dr. Rolf, o. Professor, Aloysiusstr. 28, 4630 Bochum 1, Tel. (0234) 473692

76. Grewe, Dr. Wilhelm G., o. Professor, Zum Kleinen Ölberg 28, 5330 Königswinter 41 - Thomasberg, Tel. (02244) 6874

77. Gröll, Dr. Florian, ao. Universitätsprofessor, Babenberger Str. 10, A 9020 Klagenfurt 
78. Gygi, Dr. Fritz, o. Professor, Beatusstr. 28, CH 3006 Bern, Tel. (031) 448638

79. Häberle, Dr. Peter, o. Professor, Eichleitnerstr. 30, 8900 Augsburg, Tel. (0821) 599-441 (dienstl.)

80. Häfelin, Dr. Ulrich, o. Professor, Müseliweg 1, CH 8049 Zürich, Tel. (051) 588460

81. Hahn, Dr. LL. M. Hugo J., o. Professor, Am Weinberg 7, 8700 Würzburg 25, Tel. (0931) 271904

82. Hailbronner, Dr. Kay, Professor, Am Mantelbach 6, 6901 Dossenheim, Tel. (06221) 85974 (Universität Konstanz)

83. Haller, Dr. Walter, ao. Professor, Gättenhusenstr. 8, CH 8122 Pfaffhausen-Zürich, Tel. (01) 8253671

84. Hamel, Dr. Walter, Professor, Calvinstr. 21, $3550 \mathrm{Marburg} / \mathrm{Lahn}$, Tel. (06421) 2862

85. Heckel, Dr. Martin, o. Professor, Lieschingstr. 3, 7400 Tübingen, Tel. (07071) 61427

86. Hellbling, Dr. Ernst C., o. Universitätsprofessor, Volksgasse 12, A 1130 Wien 13

87. Hengstschläger, Dr. Johann, Universitätsdozent, Auf der Halde 16, A 4020 Linz, Tel. (00437222) 31381

88. Henke, Dr. Wilhelm, o. Professor, Laufer Str. 5, 8501 Rückersdorf, Tel. (09123) 2785

(Universität Erlangen-Nürnberg)

89. Herrmann, Dr. Günter, Professor, Gregor-Vosen-Str. 8, 5042 Erftstadt-Bliesheim, Tel. (02235) 41633 (Universität Mainz)

90. Herzog, Dr. Roman, o. Professor, Ludwigstr. 35, 7024 Filderstadt 1 (Bernhausen), Tel. (0711) 705682 (Hochschule Speyer)

91. Hesse, Dr. Konrad, o. Professor, Schloßweg 29, 7802 Merzhausen üb. Freiburg/Br., Tel. (0761) 403811

92. Hettlage, Dr. Karl Maria, o. Professor, Friedrich-Ebert-Str. 83, 5320 Bad Godesberg, Tel. (02221) 364361

93. Frhr. v. d. Heydte, Dr. Friedrich August, o. Professor, Hagschneiderweg 1, 8311 Aham-Vils/Niederbayern (Universität Würzburg)

94. v. Hippel, Dr. Ernst, o. Professor, 6531 Perscheid üb. Bingen, Tel. (06744) 520 (Universität Köln) 
95. Hoegner, Dr. Wilhelm, Honorarprofessor, Am Blumengarten 17, 8000 München 9, Tel. (089) 434241

96. Hoffmann, Dr. Gerhard, Professor, Ernst-Lemmer-Str. 10/I, 3550 Marburg 6, Tel. (06421) 81645

97. Hoffmann-Riem, Dr. Wolfgang, o. Professor, Kätnerweg 24, 2000 Hamburg 65, Tel. (040) 6402478

98. Hofmann, Dr. Hasso, Wiss. Rat und Professor, Breslauer Str. 6/B, 8521 Uttenreuth, Tel. (09131) 52643 (Universität Erlangen-Nürnberg)

99. Hollerbach, Dr. Alexander, o. Professor, Parkstr. 8, 7801 March-Hugstetten üb. Freiburg/Br., Tel. (07665) 2251

100. Hoppe, Dr. Werner, o. Professor, Von-Ossietzky-Str. 41, 4400 Münster, Tel. (0251) 795500

101. Huber, Dr. Ernst Rudolf, o. Professor, In der Röte 2, 7800 Freiburg-Zähringen, Tel. (0761) 53713

102. Huber, Dr. Dr. h. c. Hans, o. Professor, Mannenriedstr. 5, CH 3074 Muri b. Bern, Tel. (031) 520925

103. Ipsen, Dr. Hans Peter, o. Professor, Haus Opferberg, 2121 Raven Post Soderstorf, Tel. (04172) 670 (Universität Hamburg)

104. Ipsen, Dr. Knut, o. Professor, Nevelstr. 59, 4630 Bochum-Weitmar, Tel. (0234) 431266

105. Isensee, Dr. Josef, o. Professor, Meckenheimer Allee 112, 5300 Bonn, Tel. (02221) 637993

106. Jaenicke, Dr. Günther, Professor, Waldstr. 13, 6906 Leimen, Tel. (06224) 3571 (Universität Frankfurt/M.)

107. Jahrreis, Dr. jur. Dr. h. c. Dr. h. c. Dr. h. c. LL. D. h. c. Hermann, o. Professor, Nasse-Str. 30, 5000 Köln 41, Tel. (0221) 413553

108. Jakob, Dr. Wolfgang, o. Professor, Wilhelmstr. 25, 8000 München 40, Tel. (089) 390506 (Universität Augsburg)

109. Jarass, Dr. Hans D., Professor, Boltzmannstr. 1, 1000 Berlin 33, Tel. (030) 8382609

110. Kaiser, Dr. jur. Dr. rer. pol. h. c. Joseph H., o. Professor, Rothofweg, 7813 Staufen, Tel. (07633) 5728 (Universität Freiburg)

111. Kewenig, Dr. Wilhelm, o. Professor, Bismarckallee 9, 2300 Kiel 1, Tel. (0431) 332322 
112. Khol, Dr. Andreas, Universitätsdozent, Jacquingasse 43, A 1030 Wien, Politische Akademie, Tel. (0222) 725186

113. Kimminich, Dr. Otto, o. Professor, Killermannstr. 6, 8400 Regensburg, Tel. (0941) 24175

114. Kipp, Dr. Heinrich, o. Universitätsprofessor, Lanserstr. 61, A 6080 Kurort Igls, Tel. (05222) 7209 (Universität Innsbruck)

115. Kirchhof, Dr. Paul, o. Professor, Südostring 37, 4401 Havixbeck, Tel. (02507) 7548 (Universität Münster)

116. Kirn, Dr. Michael, o. Professor, Rummelsburger Str. 5, 2000 Hamburg 73, Tel. (040) 6473843 (Hochschule der Bundeswehr Hamburg)

117. Kisker, Dr. Gunter, o. Professor, Waldstr. 74, 6301 Linden-Am Mühlberg, Tel. (06403) 61030 (Universität Gießen)

118. Klecatsky, Dr. Hans R., o. Universitätsprofessor, Reithmannstr. 20, A 6020 Innsbruck, Tel. (05222) 522144

119. Klein, Dr. Hans H., o. Professor, Kolberger Str. 8, 3406 Bovenden, Tel. (0551) 81704 (Universität Göttingen)

120. Kloepfer, Dr. Michael, o. Professor, Südallee 37a, 5500 Trier, Tel. (0651) 41932

121. Knemeyer, Dr. Franz-Ludwig, o. Professor, Unterdürrbacher Str. 353, 8700 Würzburg 7, Tel. (0931) 59118

122. Knies, Dr. Wolfgang, o. Professor, Sonnenweg 4, 6600 Saarbrücken 1, Tel. (0681) 54180

123. Knöpfle, Dr. Franz, o. Professor, Höhenweg 22, 8901 Leitershofen (Universität Augsburg)

124. Koch, Dr. Hans Joachim, Wiss. Rat und Professor, Wendlohstr. 80, 2000 Hamburg 61, Tel. (040) 5518804

125. Koja, D. Dr. Friedrich, o. Universitätsprofessor, Lederwaschgasse 22, A 5020 Salzburg, Tel. (06222) 222712

126. König, Dr. jur. Dr. rer. pol. Klaus, o. Professor, Wimphelingstr. 5, 6720 Speyer, Tel. (06232) 5901

127. Kopp, Dr. Ferdinand O., o. Professor, Liesel-KarlstadtStraße 24, 8033 Planegg, Tel. (089) 8599675 (Universität Passau)

128. Korinek, Dr. Karl, o. Universitätsprofessor, Auhofstr. 225-227, A 1130 Wien, Tel. (0222) 8209153

129. Krause, Dr. Peter, o. Professor, Weinbergstr. 12, 5501 Korlingen, Tel. (06588) 7333 (Universität Trier) 
130. Krawietz, Dr. Dr. Werner, Wiss. Rat und Professor, Nienbergweg 29, 4400 Münster, Tel. (0251) 53846

131. Kriele, Dr. Martin, o. Professor, Richard-Wagner-Str. 10, 5090 Leverkusen-Wald, Tel. (02172) 51564 (Universität Köln)

132. Kröger, Dr. Klaus, Professor, Hölderlinweg 14, 6300 Gießen-Wieseck, Tel. (0641) 52240

133. Krüger, Dr. Herbert, o. Professor, Philosophenweg 14, 2000 Hamburg 50

134. Küchenhoff, Dr. Erich, Wiss. Rat und Professor, Dachsleite 65, 4400 Münster, Tel. (0251) 247271

135. Küchenhoff, Dr. Günther, o. Professor, Seinsheimer Str. 13, 8700 Würzburg, Tel. (0931) 78334

136. Lange, Dr. Klaus, Professor, Klosterweg 31, 6302 Lich 6, Tel. (06404) 5681 (Universität Gießen)

137. Laubinger, Dr. Hans-Werner, Professor, Kleine Gailergasse 1, 6720 Speyer, Tel. (06232) 79678 (Universität Mannheim)

138. Lecheler, Dr. Helmut, Privatdozent, Langemarckplatz 9, 8520 Erlangen, Tel. (09131) 21789

139. Leibholz, Dr. jur. Dr. phil. Gerhard, o. Professor, Herzberger Landstr. 57, 3400 Göttingen, Tel. (0551) 57040

140. Leisner, Dr. Walter, o. Professor, Kochstr. 2, 8520 Erlangen, Tel, (09131) $87071-356$

141. Lerche, Dr. Peter, o. Professor, Junkersstr. 13, 8035 Gauting b. München, Tel. (089) 8502088

142. Link, Dr. Heinz-Christoph, o. Universitätsprofessor, Mönchsberg 17, A 5020 Salzburg, Tel. (06222) 44179

143. Lipphardt, Dr. Hanns-Rudolf, Privatdozent, Zur Forstquelle 3, 6900 Heidelberg, Tel. (06221) 382312

144. Listl, Dr. Joseph, o. Professor, Alter Postweg 120, 8900 Augsburg, Tel. (0821) 598 - 720/730 (dienstl.)

145. Lorenz, Dr. Dieter, o. Professor, Sonneneck 9, 7751 Dettingen, Tel. (07533) 6822 (Universität Konstanz)

146. Magiera, Dr. Siegfried, Privatdozent, Waitzstr. 46, 2300 Kiel, Tel. (0431) 561836

147. Mallmann, Dr. Walter, Professor, Arndtstr. 14, 6300 Gießen, Tel. (0641) 23788 
148. v. Mangoldt, Dr. Hans, Wiss. Rat und Professor, Goetheweg 1/1, 7401 Nehren, Tel. (07473) 7908 (Universität Tübingen)

149. Mantl, Dr. Wolfgang, ao. Universitätsprofessor, Wiener Str. 256/XI/33, A 8051 Graz XIII, Tel. (03122) 61306

150. Martens, Dr. Wolfgang, o. Professor, Hohenzollernring 23, 2000 Hamburg 50, Tel. (040) 8806800

151. Marti, Dr. Hans, ao. Professor, Schwanengasse 9, CH 3000 Bern, Tel. (031) 221683

152. Maunz, Dr. Theodor, o. Professor, Hartnagelstr. 3, 8032 Gräfelfing b. München, Tel. (089) 851630

153. Maurer, Dr. Hartmut, o. Professor, Säntisblick 7, 7750 Konstanz 19, Tel. (07533) 1312

154. Mayer-Tasch, Dr. Peter Cornelius, Professor, Am Seeberg 11, 8919 Schondorf am Ammersee, Tel. (08192) 668 (Universität München)

155. Meder, Dr. Walter, o. Professor, Buchsweilerstr. 20, 1000 Berlin 33, Tel. (030) 8311289

156. Meessen, Dr. Karl Matthias, o. Professor, Schillerstr. 30, 8900 Augsburg-Spickel, Tel. (0821) 555989

157. Meissner, Dr. Boris, o. Professor, Kleine Budengasse 1, 5000 Köln, Tel. (0221) 239754

158. Melichar, Dr. Dr. h. c. Erwin, o. Universitätsprofessor, Schulerstr. 20, A 1010 Wien I, Tel. (0222) 528883

159. Menger, Dr. Christian-Friedrich, o. Professor, Vredenweg 14, 4400 Münster, Tel. (0251) 86520

160. Menzel, Dr. Eberhard, o. Professor, Am See 8, 2300 Kiel-Schulensee, Tel. (0431) 65251

161. Merten, Dr. Dr. Detlef, o. Professor, Karl-Leiling-Allee 3, 6720 Speyer, Tel. (06232) 74300

162. Meyer, Dr. Hans, Professor, Klettenbergstr. 1, 6000 Frankfurt/M. 1, Tel. (0611) 552317

163. Morscher, Dr. Siegbert, Universitätsprofessor, Karl-Innerebner-Str. 88, A 6020 Innsbruck, Tel. (05222) 838333

164. Mosler, Dr. jur. Dr. jur. h. c. Hermann, o. Professor, Mühltalstr. 117, 6900 Heidelberg, Tel. (06221) 40082 
165. Müller, Dr. Friedrich, o. Professor, Von-der-Tann-Str. 15, 6900 Heidelberg 1, Tel. (06221) 31647

166. Müller, Dr. Georg, Privatdozent, Sugenreben 356, $\mathrm{CH} 5015$ Untererlinsbach (Universität Basel)

167. Müller, Dr. Jörg P., o. Professor, Kappelenring 42a, CH 3032 Hinterkappelen, Tel. (031) 360570 (Universität Bern)

168. Müller-Volbehr, Dr. Jörg, Privatdozent, Zweigstr. 170, 8031 Gröbenzell b. München, Tel. (08142) 7973

169. Münch, Dr. Fritz, apl. Professor, Zur Forstquelle 2, 6900 Heidelberg, Tel. (06221) 33599

170. v. Münch, Dr. Ingo, o. Professor, Hochrad 9, 2000 Hamburg 52, Tel. (040) 829624

171. Mußgnug, Dr. Reinhard, o. Professor, Keplerstr. 40, 6900 Heidelberg, Tel. (06221) 46222

172. v. Mutius, Dr. Albert, o. Professor, An der Krimm 10a, 6500 Mainz-Gonsenheim, Tel. (06131) 43233

173. Nicolaysen, Dr. Gert, Professor, Bockhorst 68a, 2000 Hamburg 55, Tel. (040) 8701747

174. Novak, Dr. Richard, o. Universitätsprofessor, Thadd.-Stammel-Str. 8/4, A $8020 \mathrm{Graz}$

175. Obermayer, Dr. Klaus, o. Professor, Niendorfstr. 25, 8520 Erlangen, Tel. (09131) 87606

176. Oberndorfer, Dr. Peter, o. Universitätsprofessor, Wolfauerstr. 94, A 4045 Linz, Tel. (07222) 349694

177. Öhlinger, Dr. Theo, o. Universitätsprofessor, Grenzgasse 15/16, A 1130 Wien 13

178. Oldiges, Dr. Martin, Privatdozent, Eichendorffstr. 7, 5000 Köln 30, Tel. (0221) 551082

179. v. Olshausen, Dr. Henning, Privatdozent, Am Hechenberg 22, 6500 Mainz 42, Tel. (06131) 50767

180. Oppermann, Dr. Dr. h. c. Thomas, o. Professor, Burgholzweg 122, 7400 Tübingen 1, Tel. (07071) 23762

181. Ossenbühl, Dr. Fritz, o. Professor, Im Wingert 12, 5309 Meckenheim, Tel. (02225) 2628 (Universität Bonn)

182. Papier, Dr. Hans-Jürgen, o. Professor, Neusiedler Weg 14, 4904 Enger, Tel. (05224) 5202

(Universität Bielefeld)

183. Partsch, Dr. Karl Josef, o. Professor, Lennéstr. 53 (Gartenhaus), 5300 Bonn, Tel. (02221) 655453 
184. Pernthaler, Dr. Peter, o. Universitätsprofessor, Philippine-Welser-Str. 27, A 6020 Innsbruck, Tel. (05222) 932283

185. Graf v. Pestalozza, Dr. Christian, o. Professor, Postfach 3008, 8580 Bayreuth, Tel. (0921) 608254

186. Pietzcker, Dr. Jost, Wiss. Rat und Professor, Hausdorffstr. 95, 5300 Bonn 1, Tel. (02221) $735574 / 739150$ (dienstl.)

187. Pirson, Dr. jur. Dr. theol. Dietrich, o. Professor, Moritz-von-Schwind-Str. 1, 5038 Rodenkirchen (Bez. Köln), Tel. (0221) 353328

188. Podlech, Dr. phil. Dr. jur. Adalbert, Professor, Vorm Heiligenkreuz 2, 6101 Weiterstadt, Tel. (06150) 4344 (Technische Hochschule Darmstadt)

189. Püttner, Dr. Günter, o. Professor, Georg-Hufnagel-Weg 5, 6720 Speyer, Tel. (06232) 71997

190. Quaritsch, Dr. Helmut, o. Professor, Otterstadter Weg 139, 6720 Speyer, Tel. (06232) 32637

191. Rack, Dr. Reinhard, Universitätsdozent, Obere Teichstr. 19, A 8010 Graz, Tel. (0316) 438842

192. Randelzhofer, Dr. Albrecht, o. Professor, Wulffstr. 12, 1000 Berlin 41, Tel. (030) 7926085

193. Rasenack, Dr. Christian, Professor, Dahlemer Weg 63a, 1000 Berlin 37, Tel. (030) 8173796

194. Rauschning, Dr. Dietrich, o. Professor, Rodetal 1, 3406 Bovenden, Tel. (05594) 331 (Universität Göttingen)

195. Rengeling, Dr. Hans-Werner, Wiss. Rat und Professor, Brüningheide 192, 4400 Münster, Tel. (0251) 212038 (Universität Bonn)

196. Ress, Dr. jur. Dr. rer. pol. Georg, o. Professor, Am Botanischen Garten 6, 6000 Saarbrücken, Tel. (0681) 302-3055

197. Rhinow, Dr. René A., Privatdozent, Jurastr. 48, $\mathrm{CH} 4411$ Seltisberg (Universität Basel)

198. Ridder, Dr. Helmut, Professor, Krofdorfer Str. 43, 6301 Biebertal I - Ortsteil Vetzberg, Tel. (06409) 523 (Universität Gießen)

199. Rill, Dr. Heinz Peter, o. Universitätsprofessor, Peter-Jordan-Str. 145/2, A 1180 Wien, Tel. (0222) 4757615 
200. Ringhofer, Dr. Kurt, o. Universitätsprofessor, Eduard-Macheiner-Str. 23, A 5020 Salzburg, Tel. (06222) 44767

201. Roellecke, Dr. Gerd, o. Professor, Kreuzackerstr. 8, 7500 Karlsruhe 41, Tel. (0721) 491739 (Universität Mannheim)

202. Rudolf, Dr. Walter, o. Professor, Rubensallee 55 a, 6500 Mainz-Lerchenberg, Tel. (06131) 7421

203. Rüfner, Dr. Wolfgang, o. Professor, Dreiangel 21, 2300 Altenholz ü. Kiel, Tel. (0431) 321222

204. Rühland, Dr. Curt, o. Professor, Dürerstr. 26, 3300 Braunschweig, Tel. (0531) 332116

205. Rumpf, Dr. Helmut, Honorarprofessor, Bismarckallee 27, 5300 Bonn-Bad Godesberg, Tel. (02221) 353131

206. Rupp, Dr. Hans Heinrich, o. Professor, Am Marienpfad 29, 6500 Mainz-Bretzenheim, Tel. (06131) 34588

207. Saladin, Dr. Peter, o. Professor, Forrerstr. 26, CH 3006 Bern, Tel. (031) 448006

208. Salzwedel, Dr. Jürgen, o. Professor, Siebengebirgsstr. 11, 5300 Bonn-Holzlar, Tel. (02221) 481710

209. Sasse ( $\dagger)$, Dr. Christoph, o. Professor, 3141 Echem üb. Lüneburg, Nr. 16, Tel. (040) 41234571 (dienstl.) (Universität Hamburg)

210. Sattler, Dr. Andreas, Wiss. Rat und Professor, Ludwig-Beck-Str. 17, 3400 Göttingen, Tel. (0551) 22340

211. Schäffer, Dr. Heinz, o. Universitätsprofessor, Linzer Str. 352/6/3, A 1140 Wien, Tel. (0222) 9428602 (Universität Salzburg)

212. Schenke, Dr. Wolf-Rüdiger, Wiss. Rat und Professor, Roomersheide 71, 4630 Bochum-Weitmar, Tel. (0234) 470824

213. Scheuing, Dr. Dieter H., Wiss. Rat und Professor, Meister-Johannstr. 10, 5000 Köln 41, Tel. (0221) 493399

214. Scheuner, Dr. Ulrich, o. Professor, Römerstr. 118, Appt. 2628, 5300 Bonn, Tel. (02221) 556/2628

215. Schick, Dr. Walter, o. Professor, Strindbergstr. 27, 8500 Nürnberg, Tel. (0911) 501422

216. Schiedermair, Dr. Hartmut, o. Professor, 6600 Saarbrücken, Tel. (0681) 302-3200

(Universität des Saarlandes) 
217. Schindler, Dr. Dietrich, o. Professor, Alte Landstr. 44, CH 8702 Zollikon ZH, Tel. (01) 654140 (Universität Zürich)

218. Schlaich, Dr. Klaus, o. Professor, Wolkenburgstr. 2, 5205 St. Augustin 2, Tel. (02241) 27509 (Universität Bonn)

219. Schlochauer, Dr. Hans-Jürgen, Professor, Blauenstr. 18, 6000 Frankfurt/M.-Niederrad

220. Schmid, Dr. Carlo, Professor, Raiffeisenstr. 5, 5300 Bonn (Universität Frankfurt)

221. Schmidt, Dr. Reiner, o. Professor, Bachwiesenstr. 4, 8901 Gessertshausen, Tel. (08238) 7131 (Universität Augsburg)

222. Schmidt, Dr. Walter, Professor, Senckenberganlage 31, 6000 Frankfurt/M., Tel. (0611) 7982189 (dienstl.)

223. Schmidt-Aßmann, Dr. Eberhard, o. Professor, Königsallee 63, 4630 Bochum, Tel. (0234) 34421

224. Schmidt-Jortzig, Dr. Edzard, Wiss. Rat und Professor, Maria-Montessori-Weg 11, 3400 Göttingen, Tel. (0551) 58509 (Universität Münster)

225. Schmitt Glaeser, Dr. Walter, o. Professor, Rübezahlweg 9 A, 8580 Bayreuth, Tel. (0921) 32070

226. Schnapp, Dr. Friedrich E., Wiss. Rat und Professor, Alfersheide 45, 4400 Münster, Tel. (0251) 324814

227. Schneider, Dr. Hans, o. Professor, Ludolf-Krehl-Str. 44, 6900 Heidelberg, Tel. (06221) 40381

228. Schneider, Dr. Hans-Peter, o. Professor, Delpweg 16, 3000 Hannover 91, Tel. (0511) 467166

229. Schneider, Dr. Peter, o. Professor, Goldenluftgasse 23/10, 6500 Mainz, Tel. (06131) 23273

230. Schnorr, Dr. Gerhard, o. Universitätsprofessor, Hauptstr. 10, A 6074 Rinn/Tirol (Universität Innsbruck)

231. Schnur, Dr. Roman, o. Professor, Lindenstr. 49, 7407 Rottenburg 5, Tel. (07472) 22224 (Universität Tübingen)

232. Scholler, Dr. Heinrich, apl. Professor, Zwengauer Weg 5, 8000 München 71, Tel. (089) 796424

233. Scholz, Dr. Rupert, o. Professor, Furtwänglerstr. 4, 1000 Berlin 33, Tel. (030) 8263710 dienstl.: Prof.-Huber-Platz 2, 8000 München 22, Tel. (089) 21802113 
234. Schröder, Dr. Meinhard, o. Professor, Bonner Weg 13, 5300 Bonn-Beuel 1, Tel. (02221) 440105 (Universität Trier)

235. Schwarze, Dr. Jürgen, Wiss. Rat und Professor, Ruhr-Universität Bochum, 4630 Bochum, Tel. (0234) 7005263

236. Schweitzer, Dr. Michael, Wiss. Rat und Professor, Wiesenstr. 39, 6229 Eltville - Martinsthal, Tel. (06123) 72606 (Universität Mainz)

237. Schwerdtfeger, Dr. Gunther, o. Professor, Gussau 99, 2000 Hamburg 67, Tel. (040) 6038791 (Freie Universität Berlin)

238. Scupin, Dr. Hans Ulrich, o. Professor, Robert-Koch-Str. 46, 4400 Münster, Tel. (0251) 490709

239. Seidl-Hohenveldern, Dr. Ignaz, o. Professor, Klutstein, 31, 5060 Bergisch-Gladbach, Tel. (02202) 8910 (Universität Köln)

240. Selmer, Dr. Peter, o. Professor, Nibelungenring 21, 2200 Elmshorn, Tel. (04121) 74141

(Universität Hamburg)

241. Siedentopf, Dr. Heinrich, o. Professor, Hauptstr. 170, 6741 Landau-Godramstein, Tel. (06341) 4757 (Hochschule Speyer)

242. v. Simson, Dr. Werner, o. Professor, Luisenstr. 3, 7800 Freiburg/Br., Tel. (0761) 35863

243. Skouris, Dr. Wassilios, Professor, Agio Sofias 4, Thessaloniki, Tel. (003031) 279669

244. Söhn, Dr. Hartmut, Professor, Rheinstr. 39/VI, 8000 München 40, Tel. (089) 335548 (Technische Hochschule Darmstadt)

245. Soell, Dr. Hermann, o. Professor, Domspatzenstr. 34, 8411 Etterzhausen/Nittendorf, Tel. (09404) 2125 (Universität Regensburg)

246. Spanner, Dr. Hans, o. Professor, Candidstr. 24/VII, 8000 München 90, Tel. (089) 652141

247. Staff, Dr. Ilse, Professorin, Am Forum 4, 6233 Kelkheim, Tel. (06195) 3308 (Universität Frankfurt)

248. Starck, Dr. Christian, o. Professor, Unter den Linden 20, 3400 Göttingen-Geismar, Tel. (0551) 792644

249. Steiger, Dr. Heinhard, Professor, Oberhof 16, 6300 Gießen, Tel. (0641) 74252 
250. Stein, Dr. Ekkehart, o. Professor, Jakob-Burckhardt-Str. 49, 7750 Konstanz, Tel. (07531) 63257

251. Steinberg, Dr. Rudolf, Wiss. Rat und Professor, Sextrostr. 14 B, 3000 Hannover 1, Tel. (0511) 882845

252. Steinberger, Dr. Helmut, o. Professor, Schloß West 140 , 6800 Mannheim, Tel. (06221) 36954

253. Steiner, Dr. Udo, o. Professor, Trakehner Weg 8, 4800 Bielefeld 1, (0521) 103168

254. Stern, Dr. Klaus, o. Professor, Am Stockberger Busch, 5067 Kürten, Tel. (02283) 6167 (Universität Köln)

255. Stock, Dr. Martin, Wiss. Rat und Professor, Am Knick 22, 4800 Bielefeld 1, Tel. (0521) 874643

256. Stödter, Dr. Rolf, Professor, Golfstr. 7, 2057 Wentorf b. Hamburg, Tel. (040) 7202646

257. Stolleis, Dr. Michael, o. Professor, Waldstr. 15, 6242 Kronberg 2, (Universität Frankfurt)

258. Suhr, Dr. Dieter, Wiss. Rat und Professor, Birkenstr. 39, 8900 Augsburg 22, Tel. (0821) 97646

259. Thieme, Dr. Werner, o. Professor, Am Karpfenteich 58, 2000 Hamburg 63, Tel. (040) 5384992

260. Tomuschat, Dr. Christian, o. Professor, Drachenfelsstr. 51, 5205 St. Augustin 2-Hangelar, Tel. (02241) 27541 (Universität Bonn)

261. Trzaskalik, Dr. Christoph, Privatdozent, Kisterstr. 27, 8706 Höchberg, Tel. (0931) 40124 (Universität Würzburg)

262. Tsatsos, Dr. Dimitris, o. Professor, Akademiestr. 37, Athen (Universität Thessaloniki)

263. Uber, Dr. Giesbert, o. Professor, Roseneck 5, 4400 Münster-Hiltrup, Tel. (02501) 3159

264. Ule, Dr. Carl Hermann, o. Professor, Oberer Gaisbergweg 9, 6900 Heidelberg, Tel. (06221) 27832 (Hochschule Speyer)

265. v. Unruh, Dr. Georg Christoph, o. Professor, Steenkamp 2, 2305 Kiel-Kitzeberg, Tel. (0431) 231459

266. Graf Vitzthum, Dr. Wolfgang, Professor, Prinzeneiche 11, 8130 Starnberg, Tel. (08151) 6409 (Hochschule der Bundeswehr München)

267. Vogel, Dr. Klaus, o. Professor, Ottostr. 12, 8130 Starnberg, Tel. (08151) 13221 (Universität München) 
268. Voigt, Dr. Alfred, o. Professor, Burgbergstr. 58, 8520 Erlangen, Tel. (09131) 88299

269. Wagener, Dr. Frido, o. Professor, Otto-Mayer-Str. 7, 6720 Speyer, Tel. (06232) 79165

270. Wagner, Dr. Heinz, o. Professor, Tietzenweg 54, 1000 Berlin 45

271. Wahl, Dr. Rainer, o. Professor, Sundgauallee 46, 7800 Freiburg, Tel. (0761) 85871

272. Walter, D. Dr. Robert, o. Universitätsprofessor, Iglaseegasse 72/1-2, A 1190 Wien 19, Tel. (0222) 321413

273. Wehrhahn, Dr. Herbert, o. Professor, St.-JohannesStadtwald, 6600 Saarbrücken 15, Tel. (0681) 3023188

274. Weides, Dr. Peter, o. Professor, Albertus-Magnus-Platz 1, 5000 Köln-Lindenthal 41, Tel. (0221) 4701

275. Wenger, Dr. jur. Dr. phil. Karl, o. Universitätsprofessor, Meytensgasse 18, A 1130 Wien, Tel. (0222) 8227244

276. Wengler, Dr. jur. Dr. rer. pol. Dr. jur. h. c. mult. Wilhelm, o. Professor, Werderstr. 15, 1000 Berlin 37, Tel. (030) 8016535

277. Wertenbruch, Dr. Wilhelm, o. Professor, An der Rodung 44, 5353 Katzvey (Post Mechernich), Tel. (02256) 318 (Universität Bochum)

278. Wielinger, Dr. Gerhard, Universitätsdozent, Hochsteingasse 19/29, A 8010 Graz, Tel. (03122) 648375

279. Wildhaber, Dr. Luzius, o. Professor, Gerenstr. 14, CH 3065 Bollingen/Bern, Tel. (031) 585549 (Universität Basel)

280. Wilke, Dr. Dieter, o. Professor, Thielallee 52, 1000 Berlin 33, Tel. (030) 8383011 (dienstl.)

281. Wimmer, Dr. Norbert, Universitätsprofessor, Anton-Rauch-Str. 1, A 6020 Innsbruck

282. Winkler, Dr. Günther, o. Universitätsprofessor, Reisnerstr. 22/5/11, A 1030 Wien

283. Würtenberger, Dr. Thomas, Professor, Schenkstr. 67, 8520 Erlangen, Tel. (09131) 36308 (Universität Augsburg)

284. Zacher, Dr. Hans F., o. Professor, Starnberger Weg 7, 8134 Pöcking (Universität München)

285. v. Zezschwitz, Dr. Friedrich, Professor, Petersweiher 66, 6300 Gießen, Tel. (0641) 76152 
286. Zieger, Dr. Gottfried, Professor, Leuschnerweg 10, 3400 Göttingen, Tel. (0551) 22255

287. Zimmer, Dr. Gerhard, Privatdozent, Bamberger Str. 22, 1000 Berlin 30, Tel. (030) 8544656

288. Zippelius, Dr. Reinhold, o. Professor, Niendorfstr. 5, 8520 Erlangen, Tel. (09131) 58053

289. Zuleeg, Dr. Manfred, o. Professor, Kaiser-Sigmund-Str. 32, 6000 Frankfurt 1, Tel. (0611) 564393 